\title{
Le lieu dans Un vent se lève qui éparpille : du pareil au même ?
}

\section{Suzanne Legault, Université York}

Si un thème romantique fréquent est celui du temps qui passe, le lieu où se déroule ce temps semble être à l'arrière-plan de notre imaginaire. L'univers sert de décor au récit ; il devient une toile de fond. Toutefois, la critique actuelle cherche à combler cette lacune et ne traite plus la nature physique de parent pauvre. Ainsi, André Ziethen avance que l'espace « ne se résume plus à une fonction de scène anodine sur laquelle se déploie le destin des personnages mais s'impose comme enjeu diégétique, substance générative, agent structurant et vecteur signifiant. »(\$1). Il se réfère à Jean Weisberger pour qui l'espace s'avère être « le produit d'un processus dynamique impliquant plusieurs points de vue (narrateur, personnage, lecteur) » (227).

Dans cette constellation, le rôle de l'auteur demeure bien évidemment déterminant. Dans Un vent se lève qui éparpille (1999), Jean Marc Dalpé décrit des endroits qu'il connaît car il a vécu dans la région de Sudbury pendant plusieurs années, œuvrant dans le cadre du Théâtre du Nouvel-Ontario. La notion d'espace demeure centrale dans le titre de plusieurs de ses œuvres, entre autres Les murs de nos villages (1980) et Gens d'ici (1981). Sa pièce la plus récente La Queens' (2019) se déroule aussi dans une ville ontarienne même s'il vit actuellement à Montréal. En mai 2018, à l'occasion d'une entrevue avec Catherine Perrin lors du Salon international du livre de Québec, il souligne la place cruciale qu'occupe le nord de l'Ontario dans sa création : «ce paysage-là, cette route-là, ces bars-là, [...] c'est quelque chose qui m'est resté ${ }^{1}$ ». $\mathrm{Au}$ cours de cet entretien, il acquiesce à la notion que cet espace est son centre de gravité artistique.

Puisque le lecteur est aussi appelé à jouer un rôle dans cette construction spatiale ${ }^{2}$, je précise que je suis née dans la région où ont lieu la plupart des événements de ce roman de Dalpé. La littérature que je trouvais belle, pendant ma jeunesse, se déroulait en France, voire en Russie. Selon moi, dans mon coin de pays, nous existions mais nous n'étions pas dans le narratif qui, d'office, se trouvait ailleurs. À cette époque, je n'aurais pas aimé Un vent se lève qui éparpille qui, en plus de présenter des lieux que je connais, superpose les histoires, les transformant en phénomènes à répétition dont on ne peut s'extriquer. Je lisais, moi, justement pour m'évader dans des endroits fabuleux, manoirs ou châteaux. 
Il m’est possible maintenant de mieux apprécier ce roman de Dalpé dont l'histoire se déroule dans le nord de l'Ontario. Et qui, en gros, raconte de manière décousue un inceste et un meurtre. Marie, la victime de l'inceste, et Marcel, le meurtrier de l'auteur de l'inceste, finissent par s'épouser dix ans plus tard. Ils s'établissent dans une petite ville à proximité de l'endroit où le drame a eu lieu. Dans un roman où le vent éparpille tout, les genres, les majuscules, la ponctuation, les langues, les niveaux de langue, les narrateurs, ce même vent épargne Marie et Marcel qui demeurent dans leur coin de pays alors qu'on s'attendrait à ce qu'ils quittent à la belle épouvante leur milieu. Dans cet article, je tente de donner des pistes pour répondre à la question suivante : pourquoi sont-ils restés ${ }^{3}$ ?

\section{Les genres}

Est-ce que les différents genres choisis par Dalpé proposent des réponses différentes, ce qui nous aiderait à comprendre la décision de l'auteur au sujet de l'espace qu'il octroie en fin de compte à ses personnages ?

Pourquoi accorder une telle importance aux genres ? Comme le souligne Antoine Compagnon dans l'historique qu'il trace au sujet des genres, on ne peut complètement dissocier le sens du genre : «Le genre est-il une notion transitoire ? Ou bien survit-il au sens toujours particulier auquel aboutit la compréhension ? Alors il n'est pas seulement un outil à jeter une fois que la compréhension est acquise et la compréhension est elle-même dépendante du genre (elle est genre-bound), lequel est constitutif du sens » (§2).

La frontière entre les genres ${ }^{4}$ est nettement démarquée dans ce livre qui regroupe la poésie, le roman et le théâtre. La poésie sert d'entrée en matière et se retrouve à intervalles réguliers. Des scènes de théâtre à la manière des chœurs antiques sont intercalées au fil du roman. Un des acteurs clôt l'ensemble du texte par un tonitruant « Oui, monsieur » (Le vent, $\left.189^{5}\right)$. La distinction entre les genres est respectée non seulement par la place qui leur est assignée dans le livre mais aussi par les changements de polices d'écriture.

De toute évidence, l'auteur n'a pas choisi de faire un mélange des genres comme à l'époque romantique ni, comme certains auteurs, d'intégrer pêle-mêle des genres différents dans le cadre d'un même récit. C'est plutôt un enchâssement : il octroie un espace spécifique à chacun d'entre eux pour raconter cette histoire publiée en 1999. 
Elle sera transposée sur scène en 2016 et, là encore, les genres seront délimités par une utilisation judicieuse de l'espace scénique. La poésie, chantée sur des rythmes variés, du classique au western, accapare l'arrière-scène sonore. Les personnages, ancrés par les rudiments d'une cabane de bois rond au centre de la scène, disent leur texte comme s'il s'agissait de monologues. Deux hommes en tenue campagnarde s'avancent périodiquement pour commenter ce qui vient de se passer derrière eux.

Cette présentation parallèle des trois genres est évidemment voulue et originale. Toutefois, le noyau, c'est bel et bien le récit sous forme de roman. Je commence donc par là.

\section{Le roman}

\section{A. Le rêve de l'ailleurs}

Étonnante tout de même cette décision de rester dans ce récit qui commence par le départ de Marcel pour se rendre à Toronto avec ses amis. Cette escapade lui donne l'occasion de conclure : «J't'un homme astheure ! » (28). La séance de tatouage, preuve de sa nouvelle liberté et témoignage de son amour pour Marie, l'avait de fait poussé à rêver à d'autres aventures : « il s'est imaginé assis sous un ciel tropical au bout du quai d'un port perdu du Pacifique » (24). Ce désir de l'ailleurs est partagé par nombre de personnages, entre autres, Rachel la stripteaseuse : «Ouain, m'a m'commander un tequila pis m'a faire semblant que j'suis au sacramant de Mexique ! »(150). Même par Joseph dans son camion où il retrouve «l'ancienne obsession, celle de la terre promise (plus loin, toujours plus loin) : verte vallée où vivre en paix, où l'air pur, l'eau pure et propre descendent des montagnes aux cimes blanches, étincelantes de neige et de glace » (160). Il n'y a, semble-t-il, que les Indiens au terminus pour déclarer: «We're just waiting for the train to go back home, mister » (142). Les voyages en auto, en autobus ou en camion ne sont pas synonymes de gentilles promenades ou d'excursions dans le style de Jack Kerouac. Ils visent à mettre en scène les faux départs des personnages.

En effet, le désir de l'ailleurs ${ }^{6}$ avorte malgré son intensité déjà bien nettement exprimée dans un précédent livre de Dalpé : «J'passe pas mon temps comme ceux d'icitte à parler de partir, pis à jamais me rendre plus loin que la prochaine broue. » (Le chien, 79).

B. La valise

Et pour voyager quoi de plus banal que d'emporter sa valise. La page de couverture, avec cette valise un peu vétuste, invite le lecteur à se remémorer les déplacements entraînés par le 
cours de sa propre vie. Ici il s'agit de la «vieille valise en cuir brun » (132) de Marie, valise héritée de son père. Ce dernier, mourant, avait confié sa fille à son frère Joseph et à son épouse Rose. Joseph, amoureux de sa nièce, la séduit lorsqu'elle a dix-sept-ans. Cette valise est donc lourde de souvenirs : arrivée du père de Marie, tentative de départ de Marie, objet sur lequel se focalisent les rêves érotiques de l'oncle lorsqu'il la cherche après sa fuite : «et elle tremble en déposant sa valise encore et encore, si lentement/La retrouver » (132).

Cette valise est un fil conducteur qui relie Marie à ses parents. Pour elle c'est moins un rappel de départ qu'un indicateur de l'impossible retour à sa véritable famille. Son contenu matériel n'est pas révélé mais son importance symbolique n’en est pas pour autant atténuée ${ }^{7}$ La mise en relief du rapport contenu/contenant se maintient d'ailleurs au cours du récit. Comment ignorer le coffre de cèdre de tante Rose avec les albums, les robes, les souliers blancs, coffre qu'elle étudie avec minutie, ressassant chacun des souvenirs qui l'entraîne inéluctablement vers la mort. Le coffre de tante Rose se métamorphosera en «boîte utilitaire en plastique oblongue noire » (175) qui contiendra ses cendres. Ce dernier effort pour un départ digne vers l'au-delà est presque raté car des mains de sa nièce Marie la boîte vole dans les airs ; cette dernière devra « s'agenouiller devant la boîte, se mettre à ramasser le petit tas de ce qui ne ressemble pas à des cendres mais plutôt à ces cristaux jaunasses de gros sel » (186). Sans doute vaut-il mieux que Marie, elle, n'ouvre pas sa valise de souvenirs, cette boîte de Pandore qui risque de l'empêcher de survivre. Autant ne pas regarder en arrière et risquer de subir le sort de la femme de Loth.

C. Les souvenirs

D’ailleurs, même les souvenirs ne sont pas fiables. Le narrateur omniscient du texte le proclame dès le premier chapitre : «Marcel repasse et repasse dans sa tête [...] les images qui lui restent de ce temps-là, [...] sauf qu'une bonne partie de ce qu'il croit se souvenir est inventée » (16). Et cette voix hors champ revient souvent à la charge : «Un jour, ce qu'on invente, ce qu'on fabrique, on ne se souvient plus de l'avoir fabriqué » (57). N'avait-elle pas déjà dit, cette voix, que «la mémoire n'est peut-être qu'une fiction qu'on recrée avec le peu qui nous reste, une fiction qu'on recrée au présent et pour le présent » (32) ? Ce souffleur apparemment au service d'un lecteur avide de précisions semble être soucieux de donner une forme plus consistante aux personnages. Il s'épuise à affiner les détails, à détecter les motivations secrètes, à faire des zooms dans l'espace et le temps ; c'est le meneur de jeu qui tire les ficelles des pantins qu'il ne libère jamais tout à fait. Dès le début du récit, le nombre de tirets et surtout de parenthèses ${ }^{8}$ atteste de 
ce souci, voire de ce besoin, de peaufiner chaque souvenir. Autrement, chacun d'eux risquerait de sombrer dans l'oubli et de porter atteinte à l'intégrité de cette mémoire déjà en porte-à-faux par rapport à une réalité qui se camoufle dans un jeu affolant de poupées russes. En voici un exemple :

il quittait la maison toujours avant l'aube pour ne pas être vu, n'ayant ni permis ni même l'âge de demander un permis (et même quand il avait atteint l'âge où il aurait pu demander un permis, il ne l'avait pas fait, n'en voyant pas la nécessité, ne voyant pas pourquoi il se mettrait à payer Paquette ou le gouvernement ou qui que ce soit de l'argent - qu'il n'avait pas de toute façon -, pour avoir le droit de faire ce qu'il faisait depuis des années si tout ce qu'il avait à faire c'était de ne pas être vu en train de le faire). (11-12)

Et c'est cette voix à saveur proustienne par sa méthode qui pose les jalons déterminants du roman. Les personnages ont beau se lancer dans des tentatives de monologue, le « je » de leur espace intérieur demeure fragile. Dans ces conditions, Marcel et Marie ont raison de ne pas s'éterniser sur leurs souvenirs car ils sont condamnés à s'épuiser à poursuivre des ombres chinoises. C'est dans la logique du récit tel que perçu par le narrateur omniscient.

\section{L'espace}

Les personnages surveillés par ce narrateur éprouvent en plus un attachement viscéral au cadre physique de leur milieu ${ }^{9}$. Bien qu'ils se plaignent de ce « sobre nordique plat pays blanc» (24) à la Jacques Brel, ils sont souvent sous l'emprise de cette lune mauve qui surplombe leurs activités : «il lève la tête, voit les étoiles d'un côté tandis que de l'autre, montant très haut dans le ciel, le rideau délicat, lumineux, les blanches aurores boréales dont les vastes pans semblent se mouvoir comme si on soufflait sur elles » (154). Comment concilier ces sentiments?

L'approche de Gaston Bachelard axée sur les thèmes récurrents du feu, de l'eau, de la terre et de l'air dans nombre d'œuvres littéraires est susceptible de nous orienter vers des embryons de réponses. Elle met aussi à jour des aspects importants de la dynamique du récit.

a) Le feu

Le feu, quant à lui, ne joue pas son rôle purificateur car la tentative de Marcel pour brûler la ville avorte. Il allume le feu à vingt kilomètres de la ville et en sens contraire du vent. Cet échec alimente son feu ${ }^{10}$ intérieur et favorise peut-être un passage à l'acte, le meurtre de Joseph.

b) L'eau

L'eau, en revanche, semble jouer un rôle plus positif. Pour Rose, l'eau se transforme certes en tombeau mais, pour Marcel, elle permet la cristallisation de son amour pour Marie : 
« et il se rappela la fois, l'automne dernier, quand il l'avait aperçue pieds nus dans la crique à Bissonnette » (30). Cette apparition ${ }^{11}$ qui, dans son cœur, est digne d'une Vénus de Botticelli lui permet d'échapper au contrôle du narrateur omniscient et d'affirmer: «J'vas toujours me souvenir de ça Toujours ! (31). Et grâce à la puissance mythique de cet amour, il tiendra parole n'impliquant jamais Rose dans l'assassinat de Marcel. Dans le roman, cet amour persiste. On en voit l'écho [aime] dans la première lettre des noms de la petite famille qui s'installe à Cochrane : Marcel, Marie, Méo.

c) La terre

La terre, elle, joue un rôle traditionnel : c'est ici la terre familiale, celle qu'on ne doit pas abandonner. Joseph ne peut la quitter, son père ayant déjà essuyé le refus de son plus vieux. Marcel a hérité de ce qui revenait de droit à son frère aîné mais au contraire du modèle biblique où le droit d'aînesse est usurpé; ici, on lui en fait don et cela, malgré sa réticence : « et aussitôt après le départ de son aîné, le père était venu chercher son second (lui, Joseph), l'avait obligé à se revêtir d'un manteau, à sortir dans la pluie froide qui tombait dru et à faire le tour de sa propriété [...] et durant tout le parcours l'homme avait gardé sa main rude et puissante sur la nuque de l'adolescent de quinze ans » (159). Curieusement, par un long détour, cette terre est vendue et l'argent revient à la fille du frère aîné. L'ordre social prévu est rétabli à la fin du roman.

\section{d) L'air}

Et le vent qui jouerait le rôle principal si on en croit le titre ? C'est le vent qui pousse la porte de la grange derrière Marie, le jour de leur première transgression. C'est le vent qui empêche le feu de se rendre jusqu'à la ville. C'est à ce vent, porteur du désir, que Joseph attribue un rôle dans ses fantasmes de récupération de sa nièce : « et il y aurait le vent aussi, le vent de l'ouest, printemps et sauvage qui voudrait se buter, se cogner, se ruer contre les vieilles planches grises trouées des portes et murs, qui tout d'un coup entrerait et quand il se retournerait, elle serait là » (131). C'est peut-être le vent aussi qui vide les mots de leur sens, qui les balade, qui les sépare de leur ancrage : «les minces coquilles fragiles des mots sans plus aucun poids, pour un temps s'attardant, flottant dans sa tête comme les pièces délicates d'un mobile fluet et gracile suspendu dans le vide par des fils invisibles » (95).

Pourtant, le vent n'éparpille pas Marcel et Marie. Est-ce qu'ils demeurent prisonniers de leur passé, le feu n'ayant pas joué son rôle purificateur? Ou peuvent-ils rester parce qu'ils ont 
assumé ce passé, intégré l'ambivalence essentielle au cœur des éléments, et qu'ils sont, de fait libérés? Impossible de savoir si la décision est bonne ou mauvaise.

Dans le roman, le texte se termine au cimetière. Comme le prêtre, le lecteur constate avec étonnement que Marcel, Marie et Méo sont ensemble. Et il n'y a pas de commentaire précis au sujet de l'avenir des protagonistes...

E. Le même

Mais ce qui dans la trame de ce récit est le plus déterminant, que les personnages en soient conscients ou non, c'est le thème du même. Le narrateur chuchote à l'oreille du lecteur mais aussi des personnages qu'ils se déplaceraient pour rien car tout est indifférencié.

*les villes : « aux yeux des jeunes mâles qui vivent ici comme ailleurs, dans les trous perdus [...] que la ville se nomme New York, Montréal, Buenos Aires, Manille, Naples, Shanghai, Paris (ou même Toronto), elle est toujours cette promesse envoûtante de plaisirs défendus » (19)

L'énumération est au service de l'équivalence.

*les filles : « en regardant les mêmes filles aux yeux voilés par les couches sans doute nécessaires de mauvais rye, de pot et de coke, qui prennent les mêmes poses sur les mêmes petits carrés de tapis en peau de mouton » (19-20)

La répétition rend impossible un véritable recommencement.

*les paysages : « des pins pareils à ceux qu'on retrouve sur des cartes postales dans les boutiques des hôtels au Mont Tremblant, au Colorado ou dans les Alpes » (29).

*les personnages : « la même foule depuis trois générations donc, et les mêmes personnages, elle les avait revus récemment encore, tout juste avant Noël dans ce même sous-sol, assise sur l'une des mêmes chaises de tôle grise » (68).

*les bébés : « car (mis à part les accidents, les difformes, les monstres ou les Meo) bébé naissant à l'heure du premier cri, nous nous ressemblons tous : même visage plissé, même corps crispé, même peau recouverte de sang et de placenta, même expression de terreur et d'appréhension » (87).

*les morts : « jusqu'à ce que nous nous retrouvions au bout du voyage, de nouveau pareils l'un à l'autre (même les $\mathrm{Meo}$ ) dans cette nuit commune et finale et froide » (88-89). 
*les groupes : «et depuis qu'il avait vu ce documentaire à la télévision à propos de l'exode des Palestiniens en mille neuf cents... (mais cela aurait pu tout aussi bien être celui des Rwandais ou des Éthiopiens ou des Kosovars ou des Juifs ou) » (170).

Il n'est pas nécessaire de préciser la date puisque le même destin se rejoue. Le «ou » est laissé comme une porte ouverte à tous les autres groupes qui viendront s'agglomérer au même sort.

Ce qui m'amène à conclure que, dans le roman, le projet principal n'est pas narratif. Il est plutôt d'ordre philosophique et vise à nous faire comprendre qu'il n'y a pas de nouvelles histoires. Toutes les histoires sont des variantes de la même histoire « car il n’y a rien que l'être humain aime mieux que de se faire raconter la même histoire encore et encore, que ce soit celle de Cendrillon, de Juliette ou d'Hélène de Troie... » (41).

Marcel et Marie restent peut-être tout simplement pour ne pas quitter leur zone de confort et parce que, malgré tout, ils aiment ces lieux. Le temps a aussi fait son œuvre et les gens ont sans doute commencé à oublier ce scandale. Mais la raison la plus profonde, c'est que peu importe leur choix, ils sont condamnés au même.

L'inceste les condamnait déjà à l'indifférenciation. Méo, être souffrant à l'identité instable, devient un symbole de cette transgression. Et les rituels, que ce soit pour Marcel celui de dévorer le foie de l'orignal ou encore de se faire tatouer une rose sur la poitrine lors de son voyage à Toronto, ne suffisent pas in insuffler un grand renouveau. Son lourd passé le suit.

Le « je » des personnages est prisonnier de cette araignée évoquée dès l'incipit, celle qui « se meut soudain, ses fines pattes tâtant les fils à peine visibles de la toile gluante dans laquelle s'est prise la proie qui se débat, frénétique et affolée » (9). En effet, même si le récit semble mettre en relief une ouverture de l'espace grâce à la présence fréquente de la nature extérieure, des lieux bien fermés persistent, comme celui de la prison où Marcel purge sa peine ou encore celui de la voiture où Richard Ayotte met fin au rêve d'ascension sociale d'Odette.

Dans le roman, impossible de s'en sortir car tout est semblable. C'est l'éternelle répétition.

\section{Le chœur}

C'est lors de dialogues échelonnés à la manière d'un chœur antique au cours du récit que nous, les lecteurs, apprenons les chaînons manquants grâce à des bribes de conversations entre 
deux hommes, conversations qui pourraient se dérouler au café du coin car il s'agit en grande partie des rumeurs qui se propagent sur la place publique. Nous prenons connaissance de détails révélateurs au sujet de la jeunesse difficile de Marcel, de la mort de Tante Rose, de la paternité de Méo. Même si ce chœur, écho moderne de celui de la Grèce antique, ne propose pas cinquante choreutes, il remplit bien son rôle d'informateur et de représentant de la collectivité. Il donne le mot de la fin et révèle que Marcel et Marie se sont acheté un garage dans une ville à proximité de Timmins où s'est déroulé le drame. Il commente l'ensemble du récit. Il interprète, faisant un rapprochement avec le mythe d'CEdipe ${ }^{12}$ et peut-être un clin d'œil à l'œuvre de René Girard ${ }^{13}$. Ce chœur permet à son tour une autre forme de distanciation, venant ainsi à la relève du souffleur qui se manifestait grâce aux nombreuses parenthèses au sein du récit. Elizabeth Le Corre, dans "Et nous voilà comme le chour antique : les avatars du chœur dans le théâtre de Jean Anouilh », résume cet aspect de sa contribution : "Situé en retrait de la scène, le chœur antique intervient peu dans l'action elle-même. Reflet de la communauté des spectateurs, il occupe un poste d'observateur et de commentateur expérimenté qui délivre une morale ou une sagesse universelle » $(\$ 12)$.

Le message de ces choreutes modernes rejoint celui du roman : «C'qui est grec, c'est que comme le vieux avait pas d'affaire à faire c'qu'y a fait en partant, c'est sûr que le jeune énervé va finir par faire c'qu'y a fait, pi qu'y va finir là où y'a fini. Y'a pas l'choix. Y peut penser qu'y'a un choix mais dans l'fond y'en pas » (164). Peu importe l'espace-temps, impossible de métamorphoser vraiment le kaléidoscope.

\section{Le poème}

$\mathrm{Au}$ fond, Dalpé raconte une histoire qu'il ne peut s'empêcher de rectifier à chaque tournant. Il essaie d'arriver à la vérité en donnant la parole à chaque personnage, en intervenant en tant qu'auteur omniscient, en changeant même de genre. Il soude l'ensemble par un poème qui oriente vers une plus grande abstraction. Le lecteur est entraîné du paysage aux grandes lignes, du village à la globalisation. Le «je » est devenu un «nous». Ce n’est plus uniquement l'histoire des personnages et des choreutes mais celle de «nous» tous qui « errons » dans l'espace, agités par une quête jamais assouvie, notre Big Bang s'éteignant dans le « silence minéral », dernier lieu de chaque histoire. Le premier vers de ce poème sans titre annonce le dernier vers : la nuit/le silence minéral. Voilà l'espace final où s'engouffrent les histoires. Elles 
se referment dans la nuit des temps ou tout au moins dans les roches des mines de Sudbury qui font office de caverne de Platon inversée.

Ce qui interroge constamment l'auteur, son véritable talon d'Achille, c'est l'impossibilité d'accéder au réel. Il y a toujours une « faille ». On ne s'en sort pas.

\section{La pièce de théâtre}

Et enfin, la transposition du livre sur scène ${ }^{14}$ nous oriente-t-elle vers la compréhension d'un des mobiles principaux qui sous-tendent la décision du couple ?

Si l'épine dorsale du livre se maintient, des changements dans le texte se perçoivent. Dans cette adaptation de Geneviève Parent, Johanne Melançon et Alice Ronfard, la présence de la nature du Nord ontarien s'estompe et les noms de villes ne jouent pas le même rôle, probablement pour permettre aux spectateurs de s'imprégner de l'universalité du texte. C'est aussi une manière astucieuse de transposer l'importance du même. Les acteurs parlent de manière stylisée, sans véritable interaction les uns avec les autres. Le ton désincarné rend bien l'aspect mythique mais les commentaires de quelques spectateurs à la sortie ${ }^{15}$ étaient révélateurs. Certains avaient l'impression de ne pas avoir compris le récit même s'ils avaient été séduits par la présentation moderne. L'enjeu du même, dans la représentation comme dans le livre, rend peut-être la compréhension moins aisée, d'autant plus qu'il s'ajoute aux analepses, aux prolepses, aux ellipses, aux différents genres, etc.

\section{Conclusion}

Dalpé semble rejoindre la pensée de Pierre Nepveu : «Et pourtant, le vrai départ, les voyages au bout du monde ne règlent rien, ils s'avèrent même assez décevants, car on ne quitte Kalamazoo [...] que pour constater que le monde entier ressemble à Kalamazoo » (267). En effet, Carl Sandburg dans son poème «The Sins of Karamazoo » rend compte avec sobriété et force de cette expérience : "They grow up and go to the railroad station and buy tickets for Texas, Pennsylvania, Alaska. / 'Kalamazoo is alright,' they say. 'But I want to see the world'. I And when they have looked the world over they come back saying it is all like Kalamazoo » (173).

Dans Le vent se lève qui éparpille Dalpé autorise carrément ses personnages à sauter cette étape d'errance ! Dalpé est reconnu comme auteur franco-ontarien et se reconnaît comme tel. À 
ce titre, ce récit pourrait être uniquement étiqueté comme faisant partie d'une littérature dite régionale, d'autant plus qu'il décrit fidèlement les paysages en question. Le fait de vivre dans le cadre d'un groupe minoritaire n'est certes pas anodin et, bien évidemment, le texte s'inscrit dans cette histoire collective. Cependant, tels des métalangages, les différents genres à la disposition de cet auteur poète, romancier et dramaturge, indiquent que le but constant est aussi de faire saisir au lecteur/spectateur que ce lieu, c'est tout lieu, le lieu de chacun, l'invariant de la « danse brève / obscène et sacrée » (167). Il reste à espérer que la fleur mallarméenne « l'absente de tous bouquets » (368) continue de fleurir quelque part.

\section{Bibliographie}

Bachelard, Gaston. La poétique de l'espace. Paris : PUF, 1957.

---. L'eau et les rêves. Essai sur l'imagination de la matière. Paris : Corti, 1964.

---. L'air et les songes. Paris : Corti, 1965.

Ben Jelloun, Tajar. Partir. Paris : Gallimard, 2006.

Compagnon, Antoine. Théorie de la littérature et la notion de genre, onzième leçon.

https://www.fabula.org/compagnon/genre11.php (consulté le 28 septembre 2017).

Dalpé, Jean Marc. Les murs de mon village. Sudbury : Prise de parole, 1980.

---, Gens d'ici. Sudbury : Prise de parole, 1981.

---. Le chien. Sudbury : Prise de parole, 1988.

---. Un vent se lève qui éparpille. Sudbury : Prise de parole, 1999.

---. La Queens’. Sudbury : Prise de parole, 2019.

Desbiens, Patrice. Poèmes anglais. Sudbury : Prise de parole, 1988.

Eagleton, Terry. Critique et théorie littéraires. Tr. Maryse Bouchard et Jean-François Lebouverie. Paris : PUF, coll. «Formes sémiotiques », 1994.

Entrevue. Document vidéo en ligne: https://ici.radio-canada.ca/arts/livres/rendez-vouslitteraires/evenements/document/segments/entrevue/71644/jean-marc-dalpe-defis-francoontarien (consulté le 10 janvier 2019).

Girard, René. La violence et le sacré. Paris : Grasset, 1972.

Kerouac, Jack. On the Toad. New York : Viking P, 1957. 
Le Corre, Elisabeth. "Et nous voilà comme le chœur antique: Les avatars du chœur dans le théâtre de Jean Anouilh ». https://www.erudit.org/en/journals/etudlitt/2010-v41-n1etudlitt3937/044574ar/ (consulté le 11 janvier 2018).

Mallarmé, Stéphane. « Crise de vers ». Euvres complètes. Paris : Gallimard, 1945.

Nepveu, Pierre. Intérieurs du Nouveau Monde. Montréal : Boréal, 1998.

Noël, Martine. «L'imaginaire forestier: une géocritique de trois romans franco-ontariens », thèse de maîtrise, Université d'Ottawa, 2012.

Nutting, Stéphanie et François Paré. Jean Marc Dalpé : ouvrier d'un dire. Sudbury : Institut franco-ontarien/Prise de parole, 2007.

Paré, François. Les littératures de l'exiguïté. Ottawa : Le Nordir, 1994.

Piat, Julien. « Proust par/entre parenthèse[s] ». Acta fabula 14.2, «Let's Proust again! » (2013). http://atelier.fabula.org/acta/document7587.php, (consulté le 15 juillet 2018).

Sandburg, Carl. «The Sins of Kalamazoo ». Complete Poems. Pennsylvania : The Franklin Library, special edition, 1985.

Schaeffer, Jean-Marie. Qu'est-ce qu'un genre littéraire ? Paris : Seuil, coll. « Poétique », 1989.

Serça, Isabelle. Les coutures apparentes de la recherche: Proust et la ponctuation. Paris: Champion, 2010.

Weisberger, Jean. L'espace romanesque. Lausanne : L'âge d'homme, 1978.

Ziethen, Antje. La littérature et l'espace. Arborescences 3 (juillet 2013). https://www.erudit.org/en/journals/arbo/2013-n3-arbo0733/1017363ar/ ～(consulté le 19 février 2019).

\section{Notes}

${ }^{1}$ Document vidéo en ligne. Voir la référence dans la bibliographie.

${ }^{2}$ Voir à ce sujet Terry Eagleton : «Car les lecteurs ne trouvent pas les textes dans le vide : tous les lecteurs se situent socialement et historiquement et cela influence profondément leurs façons d'interpréter les textes littéraires » (82).

${ }^{3}$ C'est d'ailleurs une question que se posent les personnages du chœur à la fin du livre : «Après tout ça, tu penserais qu'a voudrait s'éloigner le plus possible, pis surtout le plus vite possible. Tu penserais qu'une fois installée dans cette auto-là, qu'a voudrait conduire ou se faire conduire à l'autre bout du pays.» (Dalpé, Le vent, 188).

${ }^{4} \mathrm{Il}$ est entendu que les genres s'inscrivent dans une histoire synchronique et diachronique aussi bien pour l'auteur que pour les lecteurs.

${ }^{5}$ Les références à cet ouvrage seront indiquées par VL lorsque la clarté l'exige.

${ }^{6}$ Ce leitmotiv est un écho de ces vers de Patrice Desbiens dans Poèmes anglais publiés quelques années auparavant : « À Sudbury / il y a des / magasins de meubles / et des / restaurants chinois. / Il y a des maisons / et des saisons / comme partout ailleurs. / Et tout le monde vient d'ailleurs ou / veut être ailleurs. / (12-13).

${ }^{7}$ Dans son roman Partir, Tajar Ben Jelloun exploite aussi cette métaphore de la valise : « Le rêve numéro quatre est une valise, une vieille valise marron. Malika y a caché les jouets et objets qu'elle aime. Il y a de tout : une brosse à cheveux, un bout de miroir, un taille-crayon, trois boutons de couleur différente, un carnet plein de phrases écrites à 
la va-vite $[\ldots]$. Chacune de ces choses a une signification particulière. C'est son secret. Elle a juste écrit sur le dos de la valise ces mots avec un crayon feutre : "C'est à moi." » (150-151).

${ }^{8}$ Dans sa critique du livre d'Isabelle Serça au sujet de Proust, Julien Piat souligne judicieusement ce rôle des parenthèses : «d'essence digressive, la parenthèse permet de complexifier les rapports entre récit, description et commentaire : allongée, elle brouille non seulement les frontières linguistiques (entre phrase d'accueil et segment ajouté) mais encore l'organisation narrative : analepses et prolepses abondent dans ce cadre, $[\ldots] »(\S 8)$.

${ }^{9}$ Voir la thèse de Martine Noël : «La forêt du roman de Dalpé est magnétisante. [...] Les personnages sont épris de la forêt, et désirent s'y lier corps et âme afin d'en puiser la vigueur, Cependant, cette relation spirituelle ne sera pas satisfaisante ; les personnages, plus particulièrement Marcel, Joseph et Rose, ne peuvent jamais véritablement atteindre l'utopie qu'ils désirent $\gg(57)$.

10 «[...] cet étrange hurlement rauque que Marcel a poussé tout d'un coup, sorti d'on ne sait quel nœud, quel feu, quelle tumeur au ventre $[\ldots] »$ (Dalpé, VL, 46).

${ }^{11}$ Le père de Marie évoque la rencontre de sa femme en employant des termes similaires : «Est sortie d'la mer, ma belle, drette là d'vant moé. J'marchais sur la plage, j'étais perdu. Pis y'a une grosse vague qui est arrivée avec elle dessus. » (VL, 126).

12 « Tu t'envoies en l'air avec ton sang, tu finis toujours par te faire punir. » (VL, 164).

13 «Le fond de l'histoire, y's'en câlissaient! C'qu'y leur fallait à tout ce beau monde-là c't'un coupable ! » (VL, 60). Voir La violence et le sacré. La société craindrait l'indifférenciation et pour prévenir la violence que cela entraînerait, veut un coupable pour que la paix revienne.

${ }^{14}$ Création du théâtre du Nouvel-Ontario, du Théâtre de la Vieille 17 et du Théâtre français du Centre National des Arts, Un vent se lève qui éparpille, texte de Jean Marc Dalpé, mise en scène de Geneviève Pineault, saison 20162017.

${ }^{15}$ Il s'agit ici de la représentation au Théâtre français de Toronto le 3 décembre 2016. 